\title{
On the Optimum Polarizations of Incoherently Reflected Waves
}

\author{
JAKOB J. VAN ZYL, MEMBER, IEEE, CHARLES H. PAPAS, LIFE MEMBER, IEEE, AND \\ CHARLES ELACHI, SENIOR MEMBER, IEEE
}

\begin{abstract}
In radar imaging, the scattered waves are usually partially polarized. Accordingly, the concept of optimum polarization must be extended to the case of incoherent scattering where the scattered waves are partially polarized. Here, it will be shown that the Stokes scattering operator is the most suitable characterization of incoherent scattering. The problem of finding the polarization that would yield an optimum amount of power received from the scatterer is solved by assuming a knowledge of the Stokes scattering operator instead of the $2 \times 2$ scattering matrix with complex elements. The advantage of this method is that it may be used to find the optimum polarizations for the case wherein the scatterers can only be fully characterized by their Stokes scattering operator (incoherent scattering) and the case wherein the scatterer can be fully characterized by the complex $2 \times 2$ scattering matrix (coherent scattering). In this report, it will be shown that the optimum polarizations reported thas far in the literature, $i . e .$, when the problem is solved by using a knowledge of the $2 \times 2$ scattering matrix, form the solutions for a subset of a more general class of problems. When the solution of the problem is based on a knowledge of the Stokes scattering operator, it is found that for incoherent scattering six optimum polarizations can exist, whereas when the solution is based on the $2 \times 2$ scattering matrix, the number of optimum polarizations is necessarily limited to four.
\end{abstract}

\section{INTRODUCTION}

A LL HIGH-RESOLUTION radar images which have been obtained by a coherent measurement suffer from a phenomenon known as speckle noise [1]. One commonly used technique to reduce speckle noise in radar images is to add the powers received from a number of adjacent pixels for a given combination of transmit and receive polarizations. If $N$ pixels are used when the scattered powers are added, the result is a so-called $N$-look image. Since powers (rather than fields) are added, the data from the $N$ pixels are added as if the total scattering is due to $N$ scattering centers, each of which scatters incoherently. As will be shown in the next section, a combination of such scatterers cannot be fully characterized mathematically by a complex $2 \times 2$ scattering matrix. To characterize such scatterers fully, a real $4 \times 4$ matrix, the Stokes scattering operator, must be used. Generally, the resultant wave received from $N$ incoherently scattering centers is partially polarized. Moreover, the degree of polarization of the resultant wave is different for different polarizations of the wave with which the $N$ scattering centers are illuminated.

Manuscript received April 21, 1986; revised November 19, 1987. This work was supported in part by the Jet Propulsion Laboratory, California Institute of Technology, Pasadena, CA, under a contract with NASA.

J. J. van Zyl and C. Elachi are with the Jet Propulsion Laboratory, California Institute of Technology, Pasadena, CA 91109.

C. H. Papas is with the Department of Electrical Engineering, California Institute of Technology, Pasadena, CA 91125.

IEEE Log Number 8714900.
The concept of optimum polarizations in scattering problems has been discussed extensively in the literature [2]-[10]. McCormick and Hendry [10] reported results for partially polarized backscatter using the coherency matrix to characterize scatterers. Boerner et al. [5], [6], [7] mention the incoherent case and the Stokes reflection matrix (which is similar to the Stokes scattering operator). To determine the optimum polarizations in the incoherent case, they first find the elements of the scattering matrix from a knowledge of the elements of the Stokes reflection matrix and then express their result in terms of the elements of the scattering matrix. Since it is not always possible to find the elements of the scattering matrix when the elements of the Stokes scattering operator are known, this method is applicable to a very restricted class of problems.

With the increasing availability of multipolarization imaging radar data, the concept of optimum polarizations is of more than theoretical importance. Polarization has been shown to be an important tool in understanding multipolarization imaging radar results [11], [12]. For the sake of brevity only the case wherein identical antennas are used for transmission and reception will be treated here. The more general case wherein orthogonally polarized antennas or two independent antennas are used for transmission and reception may be found in [11].

\section{Characteruzation of Scatterers}

Consider the case of an antenna located at the origin of a spherical coordinate system. Also, suppose that the electric field vector of the far-zone field radiated by the antenna is $\mathbf{E}^{\mathrm{rad}}$. Now, suppose that the antenna is operated as a receiving antenna with a conjugate-matched load connected to its terminals. If a plane, monochromatic wave with an electric field vector $\mathbf{E}^{\text {inc }}$ is incident on the antenna from a direction $\theta$, $\phi$, then, in compliance with the reciprocity theorem [14], the power absorbed by the load may be written as [15], [11]

$$
P_{\mathrm{rec}}=K(\lambda, \theta, \phi)\left|\mathbf{E}^{\mathrm{rad}} \cdot \mathbf{E}^{\mathrm{inc}}\right|^{2},
$$

where

$$
K(\lambda, \theta, \phi)=\frac{\frac{1}{2}\left(\lambda^{2} / 4 \pi\right) g(\theta, \phi) \sqrt{\epsilon_{0} / \mu_{0}}}{\left|\mathbf{E}^{\mathrm{rad}}\right|^{2}},
$$

$g(\theta, \phi)$ is the antenna gain function, $\left(\lambda^{2} / 4 \pi\right) g(\theta, \phi)$ is the effective area of the antenna, and $\epsilon_{0}$ and $\mu_{0}$ are the permittivity and permeability of free space, respectively. 
Next, let an arbitrary scatterer of finite size be illuminated by a plane electromagnetic wave of infinite extent. This incident wave sets up currents in the scatterer, which in turn radiate an electromagnetic wave, the scattered wave. In the far zone of the scatterer the scattered wave is an outgoing, spherical wave.

To completely describe this scattering process mathematically, three local coordinate systems will be used. First, a Cartesian coordinate system $(x, y, z)$ is set up with its origin somewhere inside the scatterer. The transverse components of the electric field of the wave illuminating the scatterer is expressed in terms of a Cartesian coordinate system $(h, v, n)$ with its origin on the transmitting antenna. The coordinate system is set up such that the basis vector $\hat{n}$ points in the direction of propagation of the illuminating wave. The plane of incidence is defined as the plane containing $\mathbf{n}$ and the $z$-axis of the coordinate system with its origin inside the scatterer. The basis vector $\hat{\mathbf{v}}$ is orthogonal to $\hat{\mathbf{n}}$ and lies in the plane of incidence. This coordinate system is completed by requiring that the basis vector $\hat{\mathbf{h}}$ satisfies

$$
\hat{\mathbf{h}}=\hat{\mathbf{v}} \times \hat{\mathbf{n}} \text {. }
$$

The transverse components of the electric field of the scattered wave is expressed in terms of a third Cartesian coordinate system $\left(h^{\prime}, v^{\prime}, n^{\prime}\right)$ with its origin at the receiving antenna. This coordinate system is set up so that the scattered wave propagates in the negative $\hat{\mathbf{n}}^{\prime}$ direction. The basis vector $\hat{\mathbf{v}}^{\prime}$ is orthogonal to $\hat{\mathbf{n}}^{\prime}$ and lies in the plane containing both $\hat{\mathbf{n}}^{\prime}$ and the $z$-axis of the coordinate system with its origin inside the scatterer. Finally, $\hat{\mathbf{h}}^{\prime}$ is defined by

$$
\hat{\mathbf{h}}^{\prime}=\hat{\mathbf{v}}^{\prime} \times \hat{\mathbf{n}}^{\prime} \text {. }
$$

These three coordinate systems completely and unambiguously describes the scattering process mathematically. Note that this choice of the local coordinate systems ensures that the
Here, $k n-\omega t$ and $-k n^{\prime}-\omega t$ are the instantaneous phases of the illuminating and the scattered waves, respectively. Also, $n^{\prime}$ is the distance between the scatterer and the receiving antenna, $n$ is the distance between the scatterer and the transmitting antenna and the wavenumber $k$ is added in the denominator of (5) to ensure that the expression is dimensionally correct. Realizing that the electric field of the illuminating wave is the far-zone electric field radiated by the transmitting antenna $\mathbf{E}^{\mathrm{tr}}$, and using (4) and (5), it follows that one may characterize the single scatterer by a complex $2 \times 2$ scattering matrix

$$
\left(\begin{array}{l}
E_{h \prime} \\
E_{v \prime}
\end{array}\right)^{\mathrm{sc}}=\left(\begin{array}{ll}
S_{h, h} & S_{h, v} \\
S_{v, h} & S_{v, v}
\end{array}\right)\left(\begin{array}{l}
E_{h} \\
E_{v}
\end{array}\right)^{\mathrm{tr}} .
$$

In the far zone of the scatterer, the scattered wave is approximately a plane wave over the area occupied by the receiving antenna. Using this result and (6) in (1), and identifying the electric field of the wave incident on the receiving antenna $\mathbf{E}^{\text {inc }}$, as the electric field of the scattered wave $\mathbf{E}^{\text {sc }}$, and the electric field $\mathbf{E}^{\text {rad }}$ in (1) as the far-zone electric field radiated by the receiving antenna, $\mathbf{E}^{\mathrm{rec}}$, respectively, one sees that the power absorbed by the load connected to the receiving antenna is

$$
P_{\mathrm{rec}}=K(\lambda, \theta, \phi)\left|\mathbf{E}^{\mathrm{rec}} \cdot[\mathbf{S}] \mathbf{E}^{\mathrm{tr}}\right|^{2} .
$$

After some algebra it can be shown [3], [10], [11], using the right side of $(6)$, that one can write

$$
\left(\begin{array}{c}
E_{h,} \cdot E_{h^{\prime}}^{*} \\
E_{v} \cdot E_{v \prime}^{*} \\
E_{h,} \cdot E_{v+}^{*} \\
E_{v,} \cdot E_{h^{\prime}}^{*}
\end{array}\right)^{\mathrm{sc}}=[\mathbf{W}]\left(\begin{array}{c}
E_{h} \cdot E_{h}^{*} \\
E_{v} \cdot E_{u}^{*} \\
E_{h} \cdot E_{v}^{*} \\
E_{v} \cdot E_{h}^{*}
\end{array}\right)^{\mathrm{t}}
$$

where

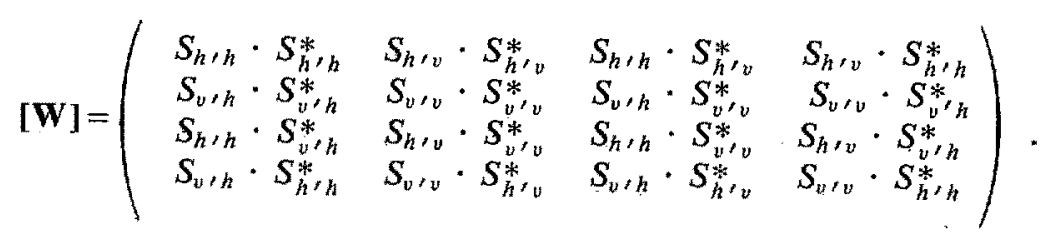

primed and unprimed coordinate systems coincide when the receiving and transmitting antennas are located at the same point, i.e., in the backscatter case. The general bistatic scattering geometry described above is shown schematically in Fig. 1.

Using local coordinate systems as shown in Fig. 1, the transverse components of the electric fields of the illuminating and scattered waves may be written as

$$
\mathbf{E}^{\mathrm{ill}}=\left[E_{\hat{h}}^{\mathrm{ill}} \hat{\mathbf{h}}+E_{v}^{\mathrm{ill}} \hat{\mathbf{v}}\right] e^{i(k n-\omega)}
$$

and

$$
\mathbf{E}^{\mathrm{sc}}=\left[E_{h^{\prime}}^{s c} \hat{\mathbf{h}}^{\prime}+E_{v^{\prime}}^{s c} \hat{v}^{\prime}\right] \frac{e^{-i\left(k n^{\prime}+\omega t\right)}}{k n^{\prime}} .
$$

If the Stokes parameters of an electric field of the form

$$
\mathbf{E}=\operatorname{Re}\left(\left[E_{\hat{h}} \hat{\mathbf{h}}+E_{v} \hat{\mathbf{v}}\right] e^{i(k r-\omega t)}\right)
$$

are defined to be

$$
\mathbf{S}(\mathbf{E})=\left(\begin{array}{c}
S_{0} \\
S_{1} \\
S_{2} \\
S_{3}
\end{array}\right)=\left(\begin{array}{l}
E_{h} E_{h}^{*}+E_{v} E_{v}^{*} \\
E_{h} E_{h}^{*}-E_{v} E_{v}^{*} \\
2 \operatorname{Re}\left[E_{h} E_{v}^{*}\right] \\
2 \operatorname{Im}\left[E_{h} E_{v}^{*}\right]
\end{array}\right)
$$

it follows that one can alternatively write (11) as

$$
\mathbf{S}(\mathbf{E})=[\mathbf{R}] \mathbf{G}(\mathbf{E})
$$


where

$$
[\mathbf{R}]=\left(\begin{array}{rrrr}
1 & 1 & 0 & 0 \\
1 & -1 & 0 & 0 \\
0 & 0 & 1 & 1 \\
0 & 0 & -i & i
\end{array}\right)
$$

and

$$
\tilde{\mathbf{G}}(\mathbf{E})=\left[E_{h} \cdot E_{h}^{*} ; E_{v} \cdot E_{v}^{*} ; E_{h} \cdot E_{v}^{*} ; E_{v} \cdot E_{h}^{*}\right] .
$$

Here, $\tilde{\mathbf{G}}$ means $\mathbf{G}$ transposed. It then follows from (12) that

$$
\mathbf{G}(\mathbf{E})=[\mathbf{R}]^{-1} \mathbf{S}(\mathbf{E}) \text {. }
$$

Using this notation, one may write (8) as

$$
\mathbf{G}\left(\mathbf{E}^{\mathrm{sc}}\right)=[\mathbf{W}] \mathbf{G}\left(\mathbf{E}^{\mathrm{tr}}\right)
$$

Now, define

$$
P_{\mathrm{rec}}^{\prime}=\left(\mathbf{E}^{\mathrm{rec}} \cdot \mathbf{E}^{\mathrm{sc}}\right) \cdot\left(\mathbf{E}^{\mathrm{rec}} \cdot \mathbf{E}^{\mathrm{sc}}\right)^{*} .
$$

Expanding the right side of (17), and using (6), one finds

$$
\begin{aligned}
P_{\mathrm{rec}}^{\prime} & =\left|E_{h}^{\mathrm{rec}} \cdot E_{h^{\prime}}^{\mathrm{sc}}+E_{v}^{\mathrm{rec}} \cdot E_{v^{\prime}}^{\mathrm{sc}}\right|^{2} \\
& =\mathbf{G}\left(\mathbf{E}^{\mathrm{rec}}\right) \cdot \mathbf{G}\left(\mathbf{E}^{\mathrm{sc}}\right) \\
& =\tilde{\mathbf{G}}\left(\mathbf{E}^{\mathrm{rec}}\right)[\mathbf{W}] \mathbf{G}\left(\mathbf{E}^{\mathrm{t}}\right) \\
& =\mathbf{S}\left(\mathbf{E}^{\mathrm{rec}}\right) \cdot[\tilde{\mathbf{R}}]^{-1}[\mathbf{W}][\mathbf{R}]^{-1} \mathbf{S}\left(\mathbf{E}^{\mathrm{tr}}\right) .
\end{aligned}
$$

If it is now defined that

$$
\begin{gathered}
\mathbf{S}^{\mathrm{rec}}=\mathbf{S}\left(\mathbf{E}^{\mathrm{rec}}\right) \\
\mathbf{S}^{\mathrm{tr}}=\mathbf{S}\left(\mathbf{E}^{\mathrm{tr}}\right)
\end{gathered}
$$

and

$$
[\mathbf{M}]=[\tilde{\mathbf{R}}]^{-1}[\mathbf{W}][\mathbf{R}]^{-1}
$$

it is clear that

$$
P_{\text {rec }}^{\prime}=\left|\mathbf{E}^{\text {rec }} \cdot \mathbf{E}^{\mathrm{sc}}\right|^{2}=\mathbf{S}^{\mathrm{rec}} \cdot[\mathbf{M}] \mathbf{S}^{\mathrm{tr}} .
$$

This $4 \times 4$ real matrix [M] will be called the Stokes scattering operator. The scattering matrix as defined in (4)-(6) describes how the scatterer transforms the illuminating electric field upon scattering. The Stokes scattering operator, on the other hand, describes how the scatterer transforms the incident Stokes parameters upon scattering. As will be shown shortly, each of these two characterizations is useful, but each can be associated with a different type of measurement.

Using the third term in (20), it is thus possible to write

$$
P_{\text {rec }}=K(\lambda, \theta, \phi) \mathbf{S}^{\text {rec }} \cdot[\mathbf{M}] \mathbf{S}^{t} .
$$

Note that (7) and (21) are identical insofar as power absorbed by the receiving antenna from a wave scattered by a single scatterer is considered.

To better understand the use of the Stokes scattering operator, consider for the moment the problem of one antenna transmitting to another and suppose that one wants to find the polarization of the transmitting antenna which would ensure that a maximum amount of power is absorbed by the load connected to the receiving antenna. From (1) it is clear that this maximum condition is

$$
\mathbf{E}^{\text {tr }}=c \mathbf{E}^{\text {rec** }}, \quad c=\text { constant } .
$$

This expression means that the receiving antenna and the wave incident on it are polarization matched if the incident wave polarization ellipse and the receiving antenna (when used as a transmitter) polarization ellipse have identical orientations in space, but the two polarization ellipses have opposite senses of rotation when both are viewed from the origin of the primed coordinate system in Fig. 1.

Now consider this same situation when the Stokes vectors are used. It is obvious that (1) may be written in the form of (7) by using a $2 \times 2$ identity matrix for [S]. If this identity matrix is used in (19), it becomes clear that (21) becomes

$$
P_{\mathrm{rec}}=K(\lambda, \theta, \phi) \mathbf{S}^{\mathrm{rec}} \cdot\left(\begin{array}{rrrr}
1 & 0 & 0 & 0 \\
0 & 1 & 0 & 0 \\
0 & 0 & 1 & 0 \\
0 & 0 & 0 & -1
\end{array}\right) \mathbf{S}^{\mathrm{tr}} \text {. }
$$

From this expression it is clear that the received power will be maximized if $\mathbf{S}^{\text {tr }}$ is identical to $\mathbf{S}^{\mathrm{rec}}$ except for a sign change in $S_{3}$. This is exactly the same as the result found above when the electric fields were used.

In the general case, there are seven independent parameters in the scattering matrix. From (7) it follows that if all the phases in [S] are referenced to the phase of one of the elements of [S], the reference phase is lost when the power absorbed is calculated. Consequently [S] contains four independent amplitudes and three independent relative phases in the general case. If no assumptions are made, this means that there must be nine relations among the 16 elements of [M]. Performing the matrix multiplications indicated in (19), it is possible to show after some tedious but straightforward algebra that these relations are [11]:

$\left(M_{11}-M_{22}\right)^{2}-\left(M_{12}-M_{21}\right)^{2}=\left(M_{33}+M_{44}\right)^{2}+\left(M_{34}-M_{43}\right)^{2}$

$M_{13} M_{23}+M_{14} M_{24}=M_{11} M_{21}-M_{12} M_{22}$

$$
M_{31} M_{32}+M_{41} M_{42}=M_{11} M_{12}-M_{21} M_{22}
$$

$M_{13} M_{14}-M_{23} M_{24}=M_{33} M_{34}+M_{43} M_{44}$

$M_{13}^{2}+M_{23}^{2}+M_{14}^{2}+M_{24}^{2}=M_{11}^{2}-M_{12}^{2}+M_{21}^{2}-M_{22}^{2}$

$M_{31}^{2}+M_{32}^{2}+M_{41}^{2}+M_{42}^{2}=M_{11}^{2}+M_{12}^{2}-M_{21}^{2}-M_{22}^{2}$

$M_{13}^{2}-M_{23}^{2}-M_{14}^{2}+M_{24}^{2}=M_{33}^{2}-M_{34}^{2}+M_{43}^{2}-M_{44}^{2}$

$M_{31}^{2}-M_{32}^{2}-M_{41}^{2}+M_{42}^{2}=M_{33}^{2}+M_{34}^{2}-M_{43}^{2}-M_{44}^{2}$.

These nine relations are necessary and sufficient conditions 


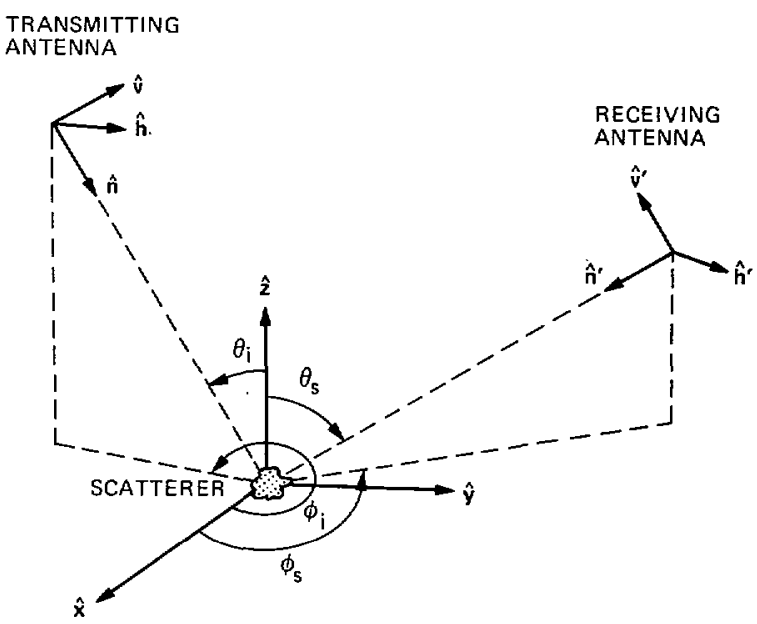

Fig. 1. Bistatic scattering geometry.

for $[\mathbf{M}]$ to be associated with a single scatterer. This means that if all these relations are true, one can always find an equivalent $2 \times 2$ (complex) scattering matrix to characterize the scatterer completely mathematically.

Now consider the case of a scatterer made up of a number of independent, incoherent scattering centers. In the case where an electromagnetic wave is a superposition of a number of electromagnetic waves with no permanent phase relationships amongst them, the Stokes parameters of the resultant wave is the sum of the Stokes parameters of the individual waves [15][18], i.e.,

$$
S_{j}=\sum_{i=1}^{N}\left(S_{j}\right)_{i}, \quad j=0,1,2,3
$$

where the index $i$ denotes each of the $N$ electromagnetic waves with independent phases. Thus, if a scatterer consists of $N$ independent, incoherent scattering centers, the Stokes. vector of the total scattered wave is

$$
\mathbf{S}^{\mathrm{sc}}=\sum_{i=1}^{N} \mathbf{S}_{i}^{\mathrm{sc}}=\left(\sum_{i=1}^{N}[\mathbf{R}][\tilde{\mathbf{R}}]\left[\mathbf{M}_{i}\right]\right) \mathbf{S}^{\mathrm{tr}},
$$

and $\left[\mathbf{M}_{i}\right]$ is the Stokes scattering operator of the $i$ th scattering center.

However, the averaging process described in (24) will not always preserve the relationships given in (22) and hence the unique connection between the scattering matrix and the Stokes scattering operator representations is lost. In such a case, no equivalent scattering matrix exists for the average Stokes scattering operator. Therefore, a scattering matrix representation will be an incomplete characterization and the Stokes scattering operator for an ensemble of incoherently scattering centers must be represented by 16 independent parameters in the bistatic case [18] and nine independent parameters in the monostatic case [4].

If a coherent measurement is made, electric fields, rather than Stokes vectors, are additive. An argument similar to the one above shows that, for a coherent measurement, the total scattering matrix is the sum of the individual scattering matrices. From (19) it follows that this total scattering matrix always has an equivalent Stokes scattering operator, even if the resultant Stokes scattering operator is not simply related to the individual Stokes scattering operators. Thus, while all scatterers can be fully characterized by a Stokes scattering operator, only coherent scattering cases can be fully characterized by a single $2 \times 2$ complex scattering matrix.

It is now evident that scatterers that can be completely characterized by a scattering matrix form a small subset of a more general class of scatterers that can be characterized fully by their Stokes scattering operator. If the problem of finding the optimum polarizations can be solved using a knowledge of the Stokes scattering operator, the solution will be applicable to a much larger class of problems than when the solution is based on a knowledge of the scattering matrix.

\section{Optimum Polarizations for Power Reception}

In the rest of this paper, it will be assumed that $\left|\mathbf{E}^{\text {tr }}\right|$ is constant for all polarizations. Also, it will be assumed that identical antennas are used for transmission and reception. Note that this assumption does not necessarily mean that the two antennas must be located at the same position in space. From (21) it then follows that one has to optimize the function

$$
P=\mathbf{S}^{\text {tr }} \cdot[\mathbf{M}] \mathbf{S}^{\text {tr }}
$$

if the power received from the scatterer is to be optimized. Assuming a normalized radiated electric field amplitude and dropping the superscript tr, one may write (25) as

$$
P(x)=\left(\begin{array}{l}
1 \\
\mathbf{x}
\end{array}\right) \cdot\left(\begin{array}{cc}
M_{11} & \tilde{\mathbf{v}} \\
\mathbf{u} & {[\mathbf{Q}]}
\end{array}\right)\left(\begin{array}{l}
1 \\
\mathbf{x}
\end{array}\right),
$$

where $x, u$, and $v$ are vectors with three coefficients and [Q] is a real $3 \times 3$ matrix. Since $\mathbf{x}$ contains the Stokes parameters of a completely polarized wave, it must satisfy the relation [15][18]

$$
\mathbf{x} \cdot \mathbf{x}=1
$$

Thus, the problem is to optimize (26) subject to the constraint condition expressed in (27). This suggests the use of Lagrange multipliers. To use the Lagrange multiplier method, the auxilliary function

$$
G(\mathbf{x})=M_{11}+\mathbf{v} \cdot \mathbf{x}+\mathbf{u} \cdot \mathbf{x}+\mathbf{x} \cdot[\mathbf{Q}] \mathrm{x}+\nu(1-\mathrm{x} \cdot \mathrm{x})
$$

is formed. Then, at the optimum values of $P(x)$, it can be shown [19] that

$$
\nabla G(\mathbf{x})=0 .
$$

Performing the indicated differentiations, one finds that the optimum polarizations have Stokes parameters which are the solutions to

$$
\left[\frac{1}{2} \mathbf{Q}+\frac{1}{2} \tilde{\mathbf{Q}}-\nu \mathbf{I}\right] \mathbf{x}=-\frac{1}{2} \mathbf{u}-\frac{1}{2} \mathbf{v},
$$

where [I] is the $3 \times 3$ identity matrix. Equation (30) represents three equations in four unknowns $\left(x_{1}, x_{2}, x_{3}\right.$, and $\nu$ ). However, the solution to (30) must also satisfy (27) [19]. Thus, the optimum polarizations are the simultaneous solu- 
tions to (27) and (30). In the next two sections, corresponding to two different cases, some ways of finding the solutions to (27) and (30) will be examined.

\section{A. $\mathbf{u}=-\mathbf{v}$}

In this case, the optimum vectors $\mathbf{x}$ are the normalized eigenvectors of $1 / 2[\mathbf{Q}+\tilde{\mathbf{Q}}]$. Since $1 / 2[\mathbf{Q}+\tilde{\mathbf{Q}}]$ is a symmetric matrix, these solutions are mutually orthogonal. The maximum (minimum) value of $P(x)$ is obtained by transmitting the eigenvector corresponding to the maximum (minimum) eigenvalue of $1 / 2[\mathbf{Q}+\tilde{\mathbf{Q}}]$.

\section{B. $\mathbf{u} \neq-\mathbf{v}$}

One possible way to find the solutions to (27) and (30) is to realize that if $\nu$ is not an eigenvalue of $1 / 2[\mathbf{Q}+\tilde{\mathbf{Q}}],(30)$ has a unique solution given by

$$
\tilde{\mathbf{x}}=-\frac{1}{2}\left[\frac{1}{2} \mathbf{Q}+\frac{1}{2} \tilde{\mathbf{Q}}-\nu \mathbf{I}\right]^{-1}(\mathbf{u}+\mathbf{v}) .
$$

To find the values of $\nu$ to use in (31), one replaces $x$ in (27) with the expression given in (31). The result is that the desired values of $y$ are found to be the real roots of

$$
p(\nu)=\nu^{6}+d_{1} \nu^{5}+d_{2} \nu^{4}+d_{3} \nu^{3}+d_{4} \nu^{2}+d_{5} \nu+d_{6}=0,
$$

where

$$
\begin{gathered}
d_{1}=-2 c_{1} \\
d_{2}=c_{1}^{2}-2 c_{2}-\frac{1}{4}(\mathbf{u}+\mathbf{v}) \cdot(\mathbf{u}+\mathbf{v}) \\
d_{3}=2 c_{1} c_{2}-2 c_{3}-\frac{1}{2}(\mathbf{u}+\mathbf{v}) \cdot\left[\mathbf{Q}+\tilde{\mathbf{Q}}-c_{1} \mathbf{I}\right](\mathbf{u}+\mathbf{v}) \\
d_{4}=c_{2}^{2}-\frac{1}{4}\left|\left[\mathbf{Q}+\tilde{\mathbf{Q}}-c_{1} \mathbf{I}\right](\mathbf{u}+\mathbf{v})\right|^{2}+2 c_{1} c_{3}-\frac{1}{2}(\mathbf{u}+\mathbf{v}) \\
\cdot\left[\frac{1}{2}(\mathbf{Q}+\tilde{\mathbf{Q}})(\mathbf{Q}+\tilde{\mathbf{Q}})-\frac{1}{2} c_{1}(\mathbf{Q}+\tilde{\mathbf{Q}})-c_{2} \mathbf{I}\right](\mathbf{u}+\mathbf{v}) \\
\left.d_{5}=2 c_{2} c_{3}-\frac{1}{2} \mathbf{I Q}+\tilde{\mathbf{Q}}-c_{1} \mathbf{I}\right](\mathbf{u}+\mathbf{v}) \cdot\left[\frac{1}{2}(\mathbf{Q}+\tilde{\mathbf{Q}})(\mathbf{Q}+\tilde{\mathbf{Q}})\right. \\
\left.-\frac{1}{2} c_{1}(\mathbf{Q}+\tilde{\mathbf{Q}})-c_{2} \mathbf{I}\right](\mathbf{u}+\mathbf{v}) \\
d_{6}=c_{3}^{2}-\frac{1}{4} \mid\left[\frac{1}{2}(\mathbf{Q}+\tilde{\mathbf{Q}})(\mathbf{Q}+\tilde{\mathbf{Q}})\right. \\
\left.-\frac{1}{2} c_{1}(\mathbf{Q}+\tilde{\mathbf{Q}})-c_{2} \mathbf{I}\right]\left.(\mathbf{u}+\mathbf{v})\right|^{2}
\end{gathered}
$$

and

$$
\begin{gathered}
c_{1}=M_{22}+M_{33}+M_{44} \\
c_{2}=\frac{1}{4}\left[\left(M_{23}+M_{32}\right)^{2}+\left(M_{24}+M_{42}\right)^{2}+\left(M_{34}+M_{43}\right)^{2}\right] \\
-M_{33} M_{44}-M_{22} M_{33}-M_{22} M_{44}
\end{gathered}
$$

$$
c_{3}=\operatorname{det}\left(\frac{1}{2}[\mathbf{Q}+\tilde{\mathbf{Q}}]\right)
$$

Here, det $(\cdot)$ means determinant of.

In general, $p(\nu)$ has at least two real roots. This can be shown by rewriting (31) in the basis formed by the eigenvectors of $1 / 2[\mathbf{Q}+\tilde{\mathbf{Q}}]$. For simplicity we shall assume that $1 / 2[\mathbf{Q}$ $+\tilde{\mathbf{Q}}]$ has three distinct eigenvalues $\lambda_{1}, \lambda_{2}$, and $\lambda_{3}$. Since this matrix is real and symmetrical, its three eigenvectors are mutually orthogonal and (31) can be written as

$$
\mathbf{x}=\left(\begin{array}{l}
b_{1} /\left(\nu-\lambda_{1}\right) \\
b_{2} /\left(\nu-\lambda_{2}\right) \\
b_{3} /\left(\nu-\lambda_{3}\right)
\end{array}\right)
$$

in the basis formed by the eigenvectors of $1 / 2[\mathbf{Q}+\tilde{\mathbf{Q}}]$. Here, we have used

$$
\frac{1}{2}(\mathbf{u}+\mathbf{v})=b_{1} \mathbf{e}_{1}+b_{2} \mathbf{e}_{2}+b_{3} \mathbf{e}_{3},
$$

and $e_{1}, e_{2}$, and $e_{3}$ are the normalized eigenvectors of $1 / 2[Q+$ $\tilde{\mathbf{Q}}$ ]. The magnitude of $\mathbf{x}$ can then be written as

$$
|\mathbf{x}|=\sqrt{\frac{b_{1}^{2}}{\left(\nu-\lambda_{1}\right)^{2}}+\frac{b_{2}^{2}}{\left(\nu-\lambda_{2}\right)^{2}}+\frac{b_{3}^{2}}{\left(\nu-\lambda_{3}\right)^{2}}} .
$$

From (35), and the assumption that at least one of the $b_{i}$ s are not zero, i.e., $\mathbf{u} \neq-\mathbf{v}$, it follows that $|\mathbf{x}|$ tends to zero as $\nu$ tends to $\pm \infty$. As $\nu$ approaches an eigenvalue, however, $|\mathbf{x}|$ approaches $\infty$. Thus, there is one value of $v$ between $-\infty$ and the smallest eigenvalue for which the corresponding $b$ is nonzero for which $|\mathbf{x}|=1$. Similarly, there is one value of $\nu$ between the largest eigenvalue for which the corresponding $b$ is nonzero and $+\infty$ for which $|\mathbf{x}|=1$. Since the roots of $p(j)$ are just the values of $\nu$ for which $|x|=1$, this proves that when $1 / 2[\mathbf{Q}+\tilde{\mathbf{Q}}]$ has three distinct eigenvalues, $p(\nu)$ has at least two real toots. Similar arguments may be used when $1 / 2[\mathbf{Q}+\tilde{\mathbf{Q}}]$ has less than three eigenvalues to show that $p(\nu)$ has at least two real roots.

Thus if $\mathbf{f}_{\mathrm{x}}$ in general, the magnitude of $\mathbf{x}$ is plotted versus $\nu$, the result will be one of the nine possibilities shown in Fig. 2 . These nine cases are possible when $1 / 2[\mathbf{Q}+\tilde{\mathbf{Q}}]$ has three distinct eigenvalues. Fig. 2(a) and (31) and (32) show that there may be a total of six optimum polarizations. Also, Fig. 2 shows that $p(p)$ always has at least two real roots as discussed above. Note that (27) and (30) and, equivalently, (31) and (32) represent the complete analytical solution to the problem at hand. Unfortunately, it is not possible to find the roots of a sixth-order polynomial analytically [20]. However, one only needs to find two real roots of $p(\nu)$ (which always exists as mentioned above). Once these two roots are known, the sixthorder polynomial can be reduced to a quartic. Many methods to find the roots of a quartic analytically are known [20].

It is not easy to show which of the six roots correspond to the maximum value of $P(x)$ and which correspond to the minimum value. However, once all the real roots are known, it is easy to find the corresponding Stokes vectors using (31). 


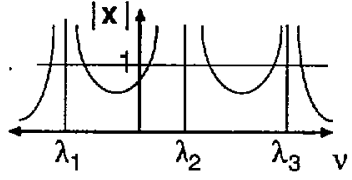

(a)

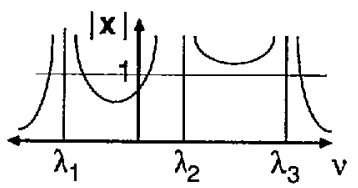

(c)

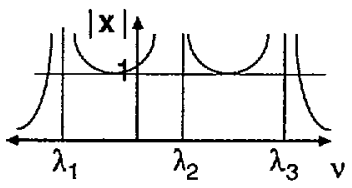

(e)

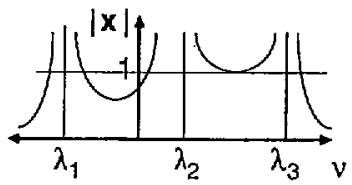

(g)

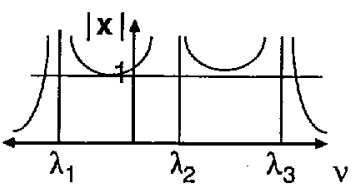

(i)

Fig. 2. General behavior of the magnitude of $\mathrm{x}$ as a function of $y$.

With all the optimum Stokes vectors known, it is little extra work to find which one corresponds to the maximum (minimum) value of $P(x)$.

In the case of incoherent backscatter, $[\mathbf{M}]$ is a symmetrical matrix [11]. Even in this case, there is no guarantee that any two solutions will be antipodal on the Poincaré sphere. If (22) is true, however, and $[\mathbf{M}]$ is a symmetrical matrix, i.e., $\mathbf{u}=$ $\mathbf{v}$, it can be shown [11] that two solutions are

$$
\mathbf{x}= \pm \frac{\mathbf{v}}{|\mathbf{v}|} ; \quad \nu=M_{11} \pm|v|
$$

These two solutions are antipodal on the Poincaré sphere. This agrees with the results found when the problem is solved assuming a knowledge of the scattering matrix.

In the case of incoherent scattering there is also no guarantee that any polarization would yield zero power absorbed by the load connected to the antenna. This, of course, is true because, in general, the scattered wave is partially polarized. The degree of polarization of the scattered wave is a function of the polarization of the incident wave:

$$
m=\frac{|\mathbf{u}+[\mathbf{Q}] \mathbf{x}|}{M_{11}+\mathbf{v} \cdot \mathbf{x}},
$$

where $m$ is the degree of polarization of the scattered wave and $\mathbf{x}$ is the Stokes vector of the incident wave.

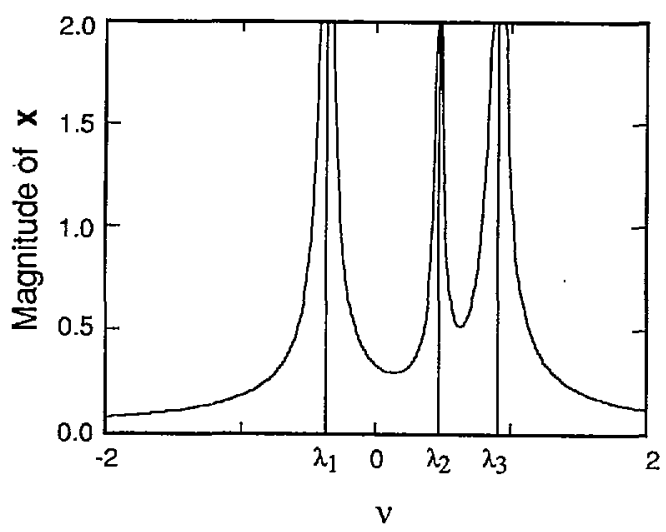

Fig. 3. $|\mathbf{x}|$ as a function of $\nu$ for the Stokes scattering operator of an urban area in San Francisco.

\section{Numerical Example}

In this section, we illustrate the optimization procedure with a numerical example. The normalized Stokes scattering operator on an urban area in San Francisco, calculated from data collected with an imaging radar polarimeter operated by the Jet Propulsion Laboratory (JPL) during the summer of 1985 [21], is

$$
[\mathbf{M}]=\left(\begin{array}{rrrr}
1.0000 & 0.0762 & 0.1399 & 0.0264 \\
0.0762 & 0.7682 & 0.3832 & -0.0615 \\
0.1399 & 0.3832 & -0.2302 & 0.0596 \\
0.0264 & -0.0615 & 0.0596 & 0.4619
\end{array}\right) \text {. }
$$

Fig. 3 shows $|\mathbf{x}|$ plotted as a function of $\nu$ for this Stokes scattering operator. The eigenvalues of $1 / 2[\mathbf{Q}+\tilde{\mathbf{Q}}]$ are

$$
\lambda_{1}=-0.3673 ; \quad \lambda_{2}=0.4655 ; \quad \lambda_{3}=0.9019 .
$$

The sixth-order polynomial for this Stokes scattering operator is found to be

$$
\begin{aligned}
p(\nu)=\nu^{6}-2 \nu^{5}+0.80901 & \nu^{4}+0.50805 \nu^{3} \\
& -0.32619 \nu^{2}-0.01404 \nu+0.02123 .
\end{aligned}
$$

Using Newton's method of successive approximations [22], the roots of this polynomial were found to be

$$
\nu=1.0163 ; 0.7864 ; 0.5145 ; 0.4171 ;-0.2619 ;-0.4725 \text {. }
$$

The optimum polarizations, with the corresponding values of the received power for this Stokes scattering operator are summarized in Table I.

Notice (Table I) that there are no optimum polarizations which are antipodal on the Poincaré sphere. Also, the minimum value of $P_{\text {rec }}$ is not zero. The polarizations corresponding to the roots $\nu=1.0163$ and $\nu=0.7864$ represent local maxima, while those corresponding to the roots $\nu=-$ 0.2619 and $\nu=-0.4725$ represent local minima of the received power. The remaining two optimum polarizations represent saddle points of the curve $P(x)$.

We also show in Table II, for comparison, the values of the received power when some commonly used polarizations are used to transmit and receive the radar waves. 
TABLE I

OPTIMUM POLARIZATIONS

\begin{tabular}{c|c|c|c|c}
\hline \multirow{2}{*}{ Root } & $P_{\mathrm{r} \varepsilon \mathrm{c}}$ & $S_{1}$ & $S_{2}$ & $S_{3}$ \\
& & & & \\
\hline & & & & \\
0.0163 & 1.6907 & 0.9190 & 0.3941 & -0.0120 \\
0.7864 & 1.5377 & -0.9522 & -0.2082 & 0.2237 \\
0.4171 & 1.3596 & -0.2632 & 0.1289 & 0.9561 \\
-.2619 & 0.8281 & -0.4165 & -0.0594 & -0.9462 \\
-.4725 & 0.4115 & 0.2404 & -0.8974 & -0.1458 \\
& & & -0.9694 & 0.0495 \\
\hline
\end{tabular}

TABLE II

RESULTS FOR SOME COMMONLY USED POLARIZATIONS

\begin{tabular}{r|r|r|r|r}
\hline Type of Polarization & $P_{\text {ree }}$ & $S_{1}$ & $S_{2}$ & $S_{3}$ \\
& & & & \\
\hline & & & & \\
Horizontal & 1.9206 & 1.0000 & 0.0000 & 0.0000 \\
Vertical & 1.6158 & -1.0000 & 0.0000 & 0.0000 \\
LH Circular & 1.5147 & 0.0000 & 0.0000 & 1.0000 \\
RH Circular & 1.4091 & 0.0000 & 0.0000 & -1.0000 \\
45 Deg. Linear & 1.0496 & 0.0000 & 1.0000 & 0,0000 \\
135 Deg. Linear & 0.4900 & 0.0000 & -1.0000 & 0.0000 \\
& & & & \\
\hline
\end{tabular}

The results in Table I show that the maximum polarization corresponds to the largest root of $p(\nu)$ and the minimum polarization corresponds to the smallest root of $p(\nu)$. Although we have observed this in all cases encountered so far, we have not been able to prove that this is always the case. This remains a topic for further study.

\section{CONCLUSION}

In this paper it is shown that a preferable way to solve for the optimum polarizations of a scatterer, is to use, as a point of departure, the Stokes scattering operator which characterizes the scatterer. The advantage of such a procedure is that it provides a solution that is applicable to both coherent and incoherent scattering problems.

\section{ACKNOWLEDGMENT}

We would like to thank our reviewers for their helpful and constructive comments.

\section{REFERENCES}

[1] F. T. Ulaby, R. K. Moore, and A. K. Fung, Microwave Remote Sensing: Active and Passive, vol. II. Reading, MA: AddisonWesley, 1981.

[2] E. M. Kennaugh, "Polarization dependence of RCS-A geometrical interpretation," IEEE Trans. Antennas Propagat., vol. AP-29, pp. 412-413, Mar. 1981 .

[3] E. M. Kennaugh, "Effects of the type of polarization on echo characteristics," Antenna Lab., Ohio State Univ., Rep. 389-9, 1951.

[4] J. R. Huynen, "Phenomenological theory of radar targets," Ph.D. dissertation, Drukkerij Bronder-Offset, N.V., Rotterdam, 1970.

[5] W.-M. Boerner, M. B. El-Arini, C.-Y. Chan, and P. M. Mastoris, "Polarization dependence in electromagnetic inverse problems," IEEE Trans. Antennas Propagat, vol. AP-29, pp. 262-270, Mar. 1981.

[6] W.-M. Boerner, "Use of polarization in electromagnetic inverse problems," Radio Sci., vol. 16, no. 6, pp. 1037-1045, Nov.-Dec. 1981.

[7] - "Optimal polarization concept in radar imaging," in Proc. ESA-EARSeL, Workshop, ESA SP-166, May 1981.

[8] H. Mieras, "Optimum polarizations of simple compound targets," IEEE Trans. Antennas Propagat, vol. AP-31, pp. 996-999, Nov. 1983.

[9] J. D. Nespor, A. P. Agrawal, and W.-M. Boemer, "Development of a model-free clutter description based on a coherency matrix formulation," in IEEE Antennas Propagat. Soc. 1984 Int. Symp. Dig., vol. I, 1984, pp. 37-40.

[10] G. C. McCormick and A. Hendry, "Optimum polarizations for partially polarized backscatter," IEEE Trans. Antennas Propagat., vol. AP-33, pp. 33-39, Jan. 1985.

[11] J. J. van Zyl, "On the importance of polarization in radar scattering problems," Ph.D. dissertation, Caltech Antenna Lab. Rep. 120, 1985.

[12] J. J. van Zyl, N. Engetha, C. H. Papas, C. Elachi, and H. Zebker, "Modelling of backscatter from vegetation layers," in 1985 Geosci. Remote Sens. Symp. Dig., vol. I, 1985, pp. 389-394.

[13] G. A. Ioannidis and D. E. Hammers, "Optimum antenna polarization for target discrimination in clutter," IEEE Trans. Antennas Propagat., vol. AP-27, pp. 357-363, May 1979.

[14] S. A. Schelkunoff and H. T. Friis, Antennas Theory and Practice. New York: Wiley, 1952.

[15] C. H. Papas, Theory of Electromagnetic Wave Propagation. New York: McGraw-Hill, 1965.

[16] M. Born and E. Wolf, Principles of Optics, sixth ed. Elmsford, NY: Pergamon, 1980.

[17] S. Chandrasekhar, Radiative Transfer. New York: Dover, 1960.

[18] H. C. van de Hulst, Light Scattering by Small Particles. New York: Dover, 1981

[19] J. A. Hummel, Introduction to Vector Functions. Reading, MA: Addison-Wesley, 1967.

[20] N. B. Conkwright, Introduction to the Theory of Equations. Boston, MA: Ginn \& Co., 1941

[21] H. A. Zebker, J. J. van Zyl, and D. N. Held, "Imaging radar polarimetry from wave synthesis," $J$. Geophys. Res, , vol. 92 , no. B1, pp. 683-701, Jan. 1986.

[22] M. Abramowitz and I. A, Stegun, Handbook of Mathematical Funciions. New York: Dover, 1965, p. 18.

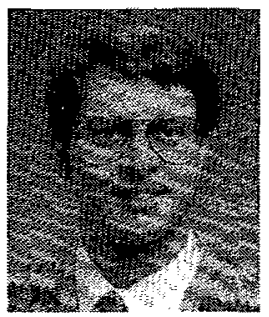

Jakob J. van Zyl (S'82-M'87) was born in Outjo, Namibia, in 1957. He received the Hons. B. Eng. degree cum laude in electronics engineering from the University of Stellenbosch, Stellenbosch, South Africa, in 1979, and also received the Siemens prize for best achievement in the graduating class from the Electrical Engineering Department, and the M.S. and Ph.D. degrees in electrical engineering from the California Institute of Technology, Pasadena, in 1983 and 1986 , respectively.

In 1984 he was the recipient of a Schlumberger Foundation fellowship. He was Teaching Assistant for a course on "Physics of Remote Sensing" at the California Institute of Technology from 1983 to 1986. In 1986 he joined the Space Sciences Division, Jet Propulsion 
Laboratory, Pasadena, CA, where he is now a Research Scientist in the Radar Sciences Group. His current interests include studying theoretical electromagnetic problems related to polarimetric scattering from rough surfaces and vegetation, remote sensing, and the analysis of polarimetric SAR data.
Verlag, 1958, with Professor Fritz Borgnis, ETH, Zurich, as co-author. He is a foreign member of the Academy of Sciences, Yerevan, USSR, and of the Academy of Sciences, Bologna, Italy.

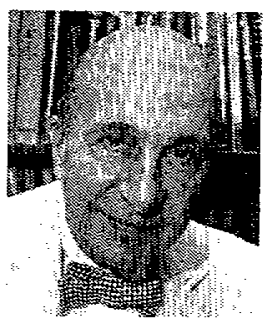

Charles H. Papas (S'41-A'42-M'55-LM'84) was born in Troy, NY, on March 29, 1918. He received the B.S. degree in electrical engineering from the Massachusetts Institute of Technology, Cambridge, MA, in 1941, and the M.S. and Ph.D. degrees from Harvard University, Cambridge, MA, in 1946 and 1948, respectively.

During the war years (1941-1945) he was with the Navy Department, Washington, DC, working at the Naval Ordnance Laboratory on the degaussing problem and at the Bureau of Ships working on microwave radar antennas. At the end of the war he returned to Cambridge to work with Professor R. W. P. King. After receiving the Ph.D. degree, he remained at Harvard University as a Research Fellow to work with Professor King on antenna and scattering problems. From 1950 to 1952 he was with the University of California as a Staff Member of the Los Alamos Scientific Laboratory, as a Consultant to the Radiation Laboratory in Berkeley, and as Lecturer in the Electrical Engineering Department, Berkeley. At the Radiation Laboratory he assisted Professor L. Alvarez in the design of a linear accelerator, and at Los Alamos he worked with Professor E. Fermi on the theory of radioflash (now known as EMP). Since 1952 he has been at the California Institute of Technology where he is Professor of Electrical Engineering and Director of the Antenna Laboratory. His research has centered on electromagnetic theory, microwaves, radio physics, gravitational electromagnetics, and basic problems in analytical electrodynamics.

Dr. Papas has written the following books: Randwertprobleme der Mikrowellenphysik, Berlin: Springer-Verlag, 1955, with Professor Fritz Borgnis, ETH, Zurich, as co-author; Electromagnetic Wave Propagation, New York: McGraw-Hill, 1965, expanded Russian edition published by Academy of Sciences, Yerevan, USSR, 1974; Lectures on Electromagnetic Theory, Academy of Sciences, Yerevan, USSR, 1973; Electromagnetic Waveguides and Resonators in Handbuch der Physik, vol. XVI, Springer-

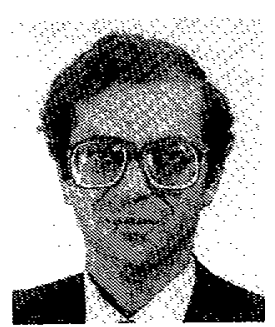

Charles Elachi (M'71-SM'82) was born in Lebanon in 1947. He received the Ingenieur degree with honors in radioelectricity and the "Prix de la Houille Blanche" from the Polytechnic Institute of Grenoble, Grenoble, France, in 1968, the B.S. degree in physics from the University of Grenoble in 1968, and the M.S. and Ph.D. degrees in electrical sciences from the California Institute of Technology, Pasadena, in 1969 and 1971, respectively.

He has worked at the Physical Spectrometry Laboratory, University of Grenoble, France, on plasma in microwave cavities. He was a Teaching Assistant at the California Institute of Technology in 1969. In 1970, he joined the Space Sciences Division, Jet Propulsion Laboratory, Pasadena, where he is presently Division Manager and a Senior Research Scientist involved in investigating spacecraft-borne scientific experiments for planetary and Earth studies using coherent radar techniques. Since 1980 , he has been the Manager for Radar Development, which covers all aspects of the radar remote-sensing program at JPL. He was the Principal Investigator on the Shuttle Imaging Radar (SIR-A) which flew in 1981, and the follow-on SIR-B experiment (1984). He is the Project Scientist of the SIR project and a Team Member on the Magellan (Venus Radar Mapper) mission. $\mathrm{He}$ has been involved in studying theoretical electromagnetic problems related to scattering from natural terrain, remote sensing, stratified media, space-time periodic media, and DFB lasers. He has 170 papers, patents, reports, and conference presentations in the above fields. He also lectures at the California Institute of Technology on the "Physics of Remote Sensing."

Dr. Elachi became the first recipient of the R. W. P. King award, in 1973. In 1980 and 1982 he received the Autometric Award of the American Photogrammetric Society. In 1982 he received the NASA Exceptional Scientific Achievement Medal and, in 1985, the W. T. Pecora award. 